\title{
A new scheme to enhance the performance of permutation index-differential chaos shift keying communications system
}

\author{
Nizar Al Bassam ${ }^{1 *}$ (1) and Oday Al-Jerew ${ }^{1,2}$
}

\author{
*Correspondence: \\ nazarhooby@yahoo.co.uk \\ ${ }^{1}$ Department of Electronics \\ and Communications \\ Engineering, Middle East \\ College, Muscat, Oman \\ Full list of author information \\ is available at the end of the \\ article
}

\begin{abstract}
In this paper, a new scheme based on permutation index-differential chaos shift keying is proposed, modeled, and evaluated in AWGN channel environment. Data is sent by frames, and each frame is headed by a single reference signal and followed by some information-bearing signals. Modulation is performed through permutations of a reference signal according to the mapped data. At the receiver, each incoming information-bearing signal undergoes all inverse permutation possibilities to perform a correlation with the delayed and stored version of the received reference signal. To decode the information bits, the detector selects the highest correlator outputs. The proposed scheme named single reference-permutation index-differential chaos shift keying is an enhanced version of PI-DCSK, and uses a single reference signal for multiple information-bearing ones. Hence, the energy requirement is saved by almost a half. The bit error performance is studied using the baseband system model and analytically tested using Gaussian approximation method. Results show the BER performance outperforms other standard and recently developed differentially coherent chaos systems, including Permutation Index-DCSK by an average of $2.25 \mathrm{~dB}$. Moreover, the analytical form which is developed to predict the bit error rate (BER) is validated by simulation. Results demonstrate the performance in AWGN is closely matching with the simulation results, particularly at high SNR.
\end{abstract}

Keywords: Differential coherence, AWGN channel, Differential correlator, Permutation index

\section{Introduction}

Chaotic communication systems are broadly classified into coherent, non-coherent and differentially coherent systems [1,2]. Implementation of coherent systems requires an accurate generation of the chaotic segment at the receiver which is identical to the transmitted one. Due to noise contribution, the received signal becomes distorted and difficult to synchronize. Theoretical implementation of coherent chaos shift keying (CSK) is proposed in [3]. Additionally, the maximum likelihood detection scheme is used in [4] to enhance bit-error-rate (BER) performance for multiple users. Changing of probability density function (PDF) for the chaotic source to match PDF of the channel to enhance BER is simulated and discussed in [5]. All the previous coherent systems require an

(c) The Author(s) 2021. Open Access This article is licensed under a Creative Commons Attribution 4.0 International License, which permits use, sharing, adaptation, distribution and reproduction in any medium or format, as long as you give appropriate credit to the original author(s) and the source, provide a link to the Creative Commons licence, and indicate if changes were made. The images or other third party material in this article are included in the article's Creative Commons licence, unless indicated otherwise in a credit line to the material. If material is not included in the article's Creative Commons licence and your intended use is not permitted by statutory regulation or exceeds the permitted use, you will need to obtain permission directly from the copyright holder. To view a copy of this licence, visit http:// creativecommons.org/licenses/by/4.0/. 
accurate regeneration of the chaotic segment at the receiver which is difficult to achieve due to the chaotic nature of the source and the channel noise. The work in $[6,7]$ considers demodulation in non-coherent systems based on the received signal properties (i.e., bit energy, map estimation, or time reversing). These systems require an effective extracting of the transmitted signal and precise threshold optimization between signal elements. In all differential chaos-based schemes, data symbols are presented by sending the reference signal followed by information bearing as in DCSK. At the receiver, correlation is performed by multiplying the incoming information-bearing signal with the delayed version of the reference signal. In correlation delay shift keying (CDSK), the transmitted signal consists of the current information signal added to the reference signal of the previous symbol as discussed in [8-11]. However, the proposed schemes are complex and require additional synchronization circuits. In DCSK, the reference signal and information-bearing signal are sent through consecutive time slots. Therefore, a frequency delay line is required at the receiver. Radio frequency (RF) delay line removal to reduce system complexity in DCSK receiver is suggested in [12] and developed in [13]. The removal is done by sending the reference signal and the information-bearing signal on the same time slot and separating them using Walsh code. Here, more delay elements are added to the transmitter in addition to the usage of lengthy Walsh code. Reference and information signal separation using initial condition modulation is examined in [14]. Efficient utilization of the transmitted signal energy using multicarrier modulation for DCSK (MC-DCSK) is introduced in [15]. However, a hardware implementation requires a bank of narrow-band modulators with a high degree of accuracy in the design to maintain subcarrier synchronization. The work in [16] uses space-time block code DCSK (STBC-DCSK) to enhance BER performance. Although STBC-DCSK enhances the noise performance of DCSK, the data rate and the average energy for each bit are similar to that in DCSK with an additional STBC encoder at the transmitter.

Permutation index DCSK is used to build the first MARY modulation scheme in [17, 18], and an advanced version of the PI-DCSK is proposed on [19]. Improved versions of PI-DCSK where reference chaotic segment and information-bearing signal are combined on one-bit duration are presented in [20]. Designing a chaotic communications system using a multilevel (MARY) approach is also proposed in [21]. The aim of the proposed system is to increase the throughput with the ability to operate at high carrier frequencies, low-cost, low-power consumption, and a simple circuit. Walsh Code is also recalled in [22] to separate reference and information-bearing signal. While the dual used mode of the reference signal is being suggested in [23]. In all the previous literature, DCSK is based on having one to one relationship between the reference signal and information-bearing signal. In this brief, a common reference signal is used to modulate multiple information bits respective by signal permutation according to the information bits, hence the proposed system enhances the energy efficiency by almost $50 \%$. Also, it offers double bit rates compared to standard chaos-based systems, namely DCSK, Correlation Delay Shift Keying, high efficiency-DCSK (HE-DCSK), and time reversal-DCSK (TR-DCSK).

Many communication schemes and applications have been developed and designed for chaos-based communication systems where energy, data rate, and synchronization awareness were the focus of the design. The work in [24] proposed various 
methodologies to improve the quality of the chaotic signal and to guarantee a high level of security and randomness. Synchronization between two matching chaotic systems to provide confident communication is considered in [25]. The work in [26] presents the generation of random number and a chaos-key that is suitable for data encryption applications. Using segmented logistic map to improve the chaotic map in the design of chaotic encryption algorithm is proposed in [27], while the work in [28] uses a neural network chaos algorithm for the development of dynamic key encryption and decryption algorithms for wireless communication security. The position of nodes in wireless sensor networks is estimated using discrete chaotic mapping in [29]. A software-defined radio system is used in [30] to study the behavior of chaotic sequences in a spreadspectrum system and show that these sequences can be used as spreading sequences in spread spectrum systems. Using the field programmable gate arrays (FPGAs) to implement the synchronization between two chaotic systems with heterogeneous dynamics was the focus in [31]. There are several other wireless applications that use non-coherent chaos-based communication such as multiple antennas [32], cooperative networks, network coding and power line communications [33].

The main contribution of this paper is as follows: we develop an SR-PI-DCSK which uses a single reference signal for multiple information-bearing ones. We compare BER performance with the other differentially and permutation-based DCSK that uses a dedicated reference signal for each information symbol. The developed model is mathematically analyzed and verified by simulation under various factors and different channel conditions.

The remainder of this paper is organized as follows. Sections 2 and 3 present the transmitted signal frame and permutation process, respectively. Section 4 describes the scheme for the proposed SR-PI-DCSK system. The details of system analysis are discussed in Sects. 5 and 6. The proposed system complexity analysis is evaluated in Sect. 7. The details of experimental results with discussion are provided in Sect. 8. Finally, Sect. 9 concludes the paper.

\section{System signal format}

The transmitted signal frame of SR-PI-DCSK is shown in Fig. 1. Each frame consists of a reference signal segment with a length of $\beta$ samples followed by $L$ information-bearing signals. Information-bearing signal is nothing but only a permutated version of the reference signal. Permutation order is determined by the information bits with the length

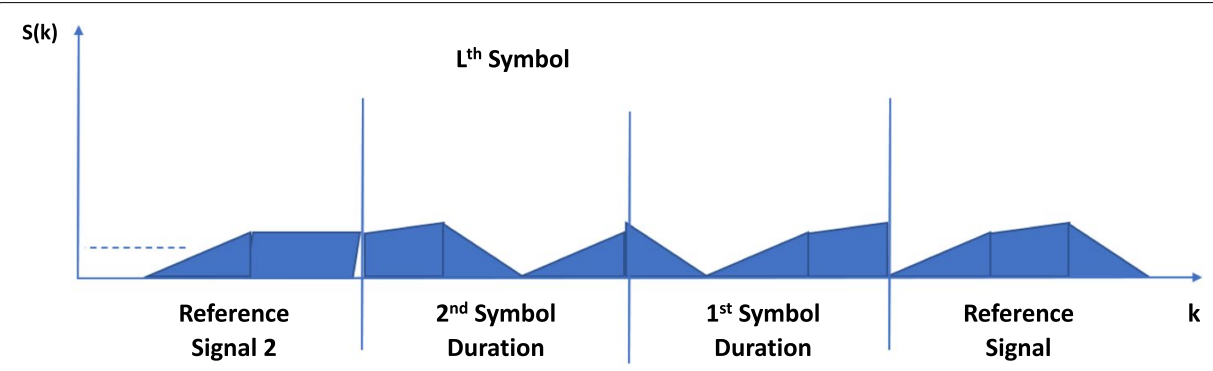

Fig. 1 SR-PI-DCSK transmitted signal frame 
of $n$, for example, to send 2 information bits, we need to set up $2^{2}$ permutations from the reference signal while 3 bits require 9 different permutations.

\section{Permutation process}

Changing of samples order in each chaotic segment is achieved using permutation process. The reordering process is determined by the permutation matrix. The permutation matrix $P_{j 1}$ is square matrix with dimension equal to the chaotic segment length. A chaotic signal with the spreading factor of $\beta$ can be permutated in $\beta !-1$ permutation. Hence for information bits vector $a=\left(\begin{array}{llll}a_{1} & a_{2} & \ldots & a_{n}\end{array}\right)$ with length of $n$, we will select one distinguished permutation out of $\beta !-1$ and to have permutation mapping such away that $a_{j} \rightarrow P_{j}$ and $P_{j} \in\left\{P_{1} P_{2} \ldots P_{2^{n}}\right\}$. For example, if $X_{0}=x_{1}, \ldots, x_{4}$, and the permutation matrix is

$$
P_{j 1}=\left[\begin{array}{llll}
0 & 1 & 0 & 0 \\
0 & 1 & 0 & 0 \\
0 & 0 & 1 & 0 \\
1 & 0 & 0 & 0
\end{array}\right]
$$

Then the permutated output can be written as $X_{0} P_{j 1}=x_{4}, x_{2}, x_{3}, x_{1}$. Furthermore, $P_{j}$ is the permutation performed in the chaotic sequence to present $a_{j}$ data set.

\section{The proposed scheme}

To generate the signal in Fig. 1, the design of modulator and demodulator of SR-PIDCSK system is shown in Figs. 2 and 3, respectively. A reference signal $X$ is generated with the chaotic samples having a length of $\beta$. This reference signal is sent initially prior to the frame through switch $S$, to modulate the first information symbol, the same version of the reference signal is delayed and undergoes permutation operation according to the transmitted bits. The reference signal and its permutated version are related in a way that ensures very low correlation such that $X P_{j}^{T}(X)=0$ [17], where $P_{j}($.$) is the per-$ mutation operator.

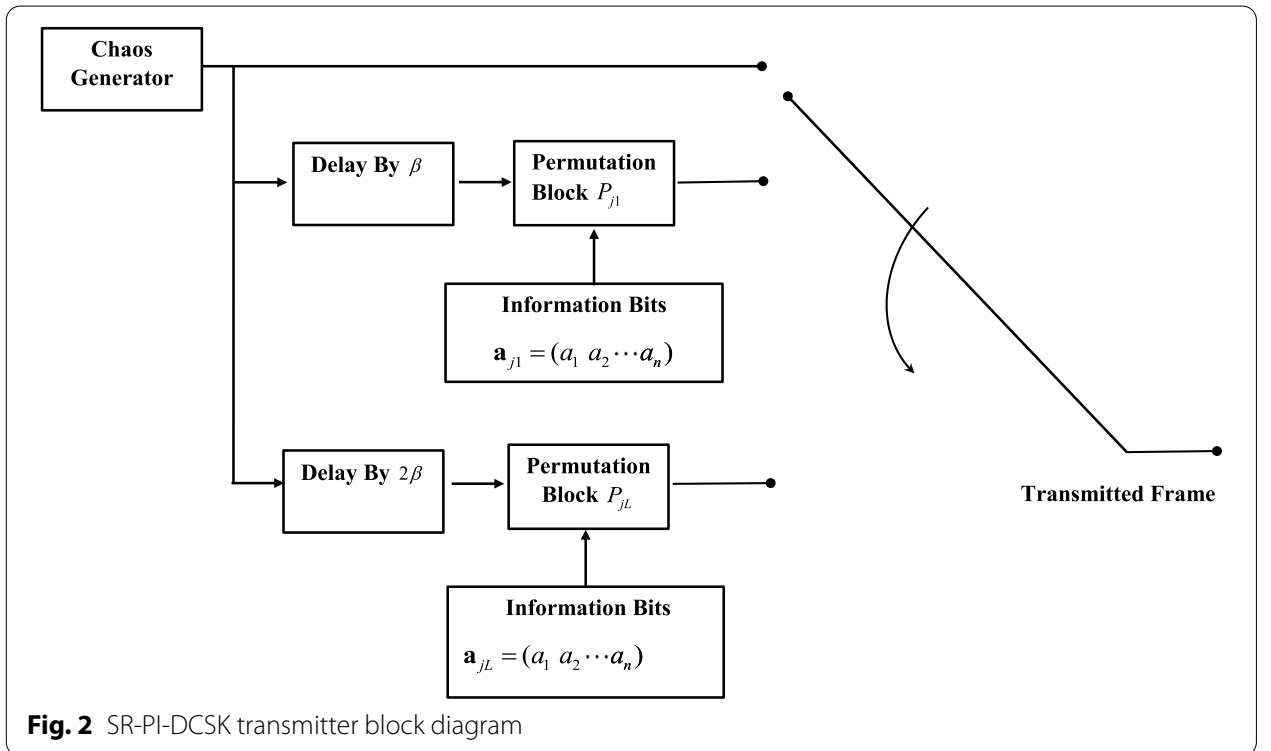




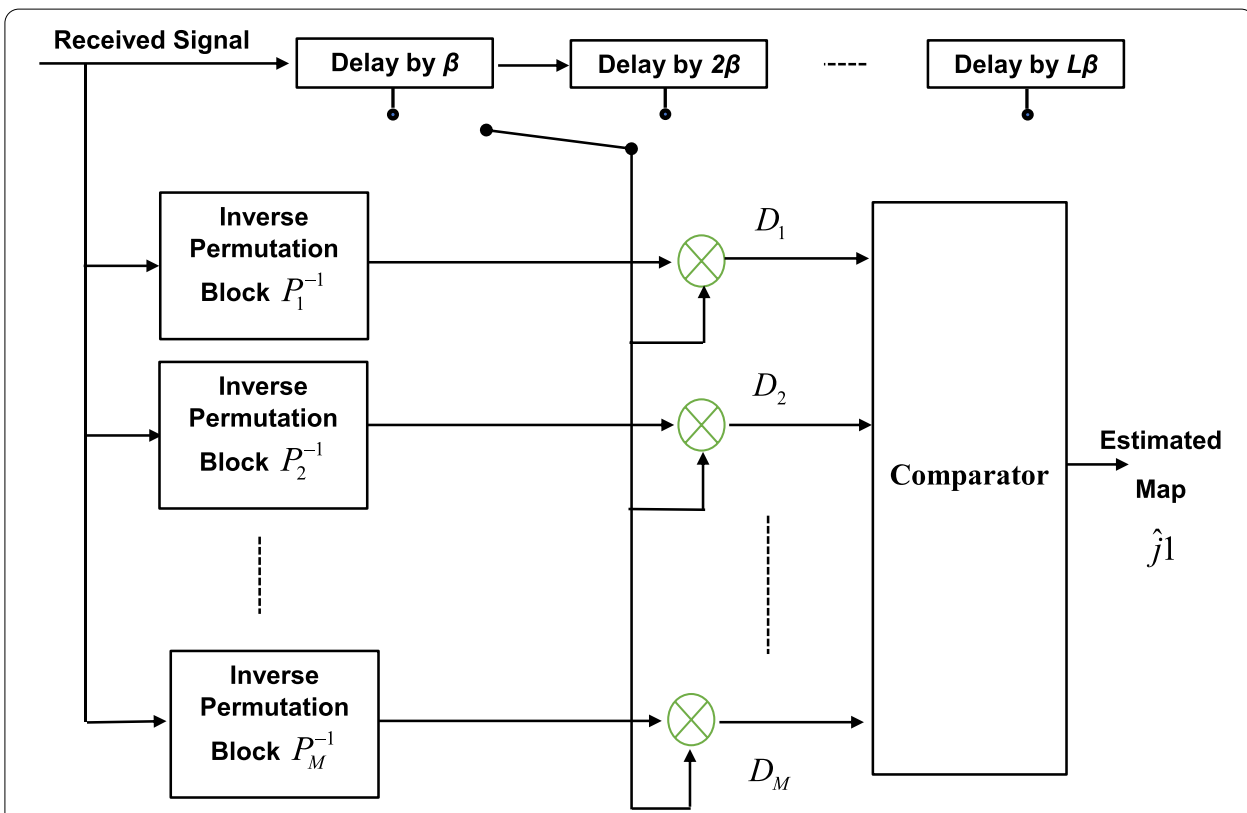

Fig. 3 SR-PI-DCSK receiver diagram

Thus, the first transmitted frame can be given by the baseband representation as:

$$
S=\left[\begin{array}{lll}
\underbrace{X_{0}}_{\text {reference }} & \underbrace{P_{j 1} X_{\beta}^{T}}_{1 s t \text { symbol }} & \underbrace{P_{j 2} X_{2 \beta}^{T}}_{\text {2nd symbol }} \cdots \underbrace{P_{j L} X_{L \beta}^{T}}_{\text {Lth Symbol }}
\end{array}\right]
$$

where $X_{0}=\left(x_{1} x_{2} \ldots x_{\beta}\right)$ is the reference chaotic segment and $P_{j 1} X_{\beta}^{T}$ represents the first information signal which is nothing but reference signal delayed by $\beta$ and permutated by $P_{j 1}$ mapped from information vector $a_{j 1}$. Moreover, $L$ is the frame length. In this paper, the analysis is simplified using the analysis of the first symbol only, similar methods can be applied on other following information symbols since all the symbols are statistically independent.

Under the assumption that the transmitted signal is received via AWGN channel and the transmitter and receiver are perfectly synchronized. The following operations are performed in the receiver: (1) The first incoming reference signal, is loaded into delay block and stored for correlation with other $L$ symbol as shown in Fig. 2. (2) The following information-bearing signal is directly loaded to the permutation block to perform all possible $M$ inverse permutations, where $M=2^{n}$.

To detect the first information symbol, the received reference signal is delayed by $\beta$, (i.e., $X_{\beta}+W_{\beta}$ ) is correlated with output of $m^{\text {th }}$ inverse permutation block output where $m \in(1,2, \ldots, M)$, hence, the output can be written as $P_{m}^{-1}\left(P_{j 1} X_{\beta}+W_{0}\right)$ where $W_{0}=\left(w_{1} w_{2} \ldots w_{\beta}\right)$ is the noise vector and $w_{k}$ is AWGN noise sample having power spectral density of $N_{0} / 2$. Therefore, the $m^{\text {th }}$ correlator output can be found as 


$$
\begin{aligned}
D_{m} & =\left(X_{\beta}+W_{\beta}\right) P_{m}^{-1}\left(P_{j 1} X_{\beta}+W_{0}\right) \\
& =\underbrace{X_{\beta} P_{m}{ }^{-1} P_{j 1} X_{\beta}^{T}}_{S S \text { or SI }}+\underbrace{X_{\beta} W_{0}^{T}+W_{\beta} P_{m}{ }^{-1} P_{j 1} X^{T}}_{S N}+\underbrace{W_{\beta} P_{m}{ }^{-1} W_{0}^{T}}_{N N}
\end{aligned}
$$

where $S S$ represents desired signal component at $m=j_{1}$, while $S I$ is the inter signal interference components at $m \neq j_{1}$. Inter signal interference results from the correlation between the chaotic signal and its permutated version. The $N N$ is the noise to noise correlation that has a significant impact on the performance particularly at a large spreading factor of $\beta$.

The first estimated permutation index $\hat{j}_{1}$ is determined by correlator which is having the maximum output compared with the others, hence

$$
\hat{j}_{1}=\arg \max \left\{\mathrm{D}_{m}\right\}, m \in\left\{1,2, \ldots 2^{n}\right\}
$$

By estimating the correct $\hat{j}_{1}$, the transmitter can predict which permutation which has been used to send the information.

Table 1 compares the proposed system with other chaos-based systems, clearly, the proposed scheme offers several features compared with DCSK, CDSK, HE-DCSK, and TR-DCSK. With respect to energy per bits SR-PI-DCSK, the requirement is significantly reduced. This is due to the use of a single reference signal in each frame. Moreover, the proposed scheme offers superior spectral efficiency as each information-bearing signal is mapped with B bits and this reference is used multiple times in each frame. This arrangement guarantees spectral efficiency with respect to DCSK, CDSK, and HE-DCSK. On the other hand, reasonable system complexity is needed to achieve these features.

\section{System analysis}

The BER performance analysis is performed analytically using GA methods that are suitable for a system having large spreading factors. However, for low spreading factors, an accurate integration method is suggested [10].

For each $L$ symbols sent, one reference signal is dedicated; therefore, $L E_{\text {symbol }}=(L+1) E_{s}$, where $E_{\text {symbol }}$ is the average symbol energy while $E_{s}$ is average segment energy given by $\beta V(X)$ and $V($.$) is the variance operator. For standard DCSK and$ CDSK and standard scheme [18-20, 22, 23], each information-bearing signal is accompanied by reference signal. Therefore, to send $L$ symbols, we need to have $L$ additional

Table 1 Chaos-based communication systems comparison

\begin{tabular}{lllllll}
\hline System & Energy saving & $\begin{array}{l}\text { Spectral } \\
\text { efficiency }\end{array}$ & Security level & $\begin{array}{l}\text { Reference } \\
\text { signal } \\
\text { required }\end{array}$ & $\begin{array}{l}\text { Permutation } \\
\text { required }\end{array}$ & $\begin{array}{l}\text { Mode of bit } \\
\text { transmission } \\
\text { for each } \\
\text { reference } \\
\text { signal }\end{array}$ \\
\hline SR-PI-DCSK & High & High & High & No & Yes & Multiple \\
DCSK & No & Low & Low & Yes & No & Single \\
CDSK & No & Low & Moderate & Yes & No & Single \\
HE-DCSK & Moderate & Low & Moderate & Yes & No & Dual \\
TR-DCSK & Moderate & Moderate & Moderate & Yes & Yes & Single
\end{tabular}


reference signal, hence energy and bandwidth efficiency are almost doubled. Compared with the proposed scheme, and for sufficient value of $L$, the energy efficiency can be calculated as

$$
E_{\text {efficiency }}=\frac{L+1}{2 L} \approx 50 \% \text { for sufficient value of } L
$$

Since each symbol is carrying $n$ bits, hence average bit energy is given by

$$
\begin{aligned}
& L E_{\text {symbol }}=L n E_{b} \\
& \frac{(L+1)}{L n} \beta V(X)=E_{b}
\end{aligned}
$$

\section{Theoretical performance analysis}

In this section, the performance analysis of the proposed scheme is analyzed in AWGN channel environment to predict the BER performance. The chaotic sequence is generated from the symmetric Tent map, given by the equation $x_{k+1}=1-2\left|x_{k}\right|$. The chaotic sequence $x$ is having uniform distribution between -1 and 1 with zero mean and computed variance $V(x)=1 / 3$ and $V\left(x^{2}\right)=4 / 45=4 / 5 V^{2}(x)$ [11]. For the rest of the analysis, the following assumptions are considered (1) The correlation between the chaotic sequence and its permutated version is decaying quickly for a sufficient correlation window. (2) The correlation between the chaotic sequence and the noise sample is statistically independent.

The Bit Error Probability of the proposed scheme is determined by calculating (1) erroneous permutation estimation $P r_{\text {map }}$, (2) erroneous probability of bits detection which largely depends on the number of bits $n$ which calculated by the following

$$
P_{r_{\text {map }}}=\frac{2^{(n-1)}}{2^{n}-1} P_{r_{\mathrm{ed}}}
$$

Correct estimation of the transmitted sequence is done by selecting the maximum absolute value of one of the correlator outputs. This output results from the correlation between the delayed reference signal and inverse permutated information-bearing signal. Each correlator output can be modeled as a Gaussian random variable $D_{m}$. For equiprobable transmitted sequence, the error probability of permutation index estimation conditioned by $P_{j}$ is given by

$$
P_{\text {red }}=\left(P_{r}\left|D_{j 1}\right|<\max P_{r}\left|D_{m}\right|\right) \text { for } 1 \leq m \leq 2^{n}, m \neq j_{1}
$$

where the $D_{j 1}$ and $D_{m}$ are decision variable at $m$ th and $j_{1}^{\text {th }}$ correlator output. The error will occur only if any value of $D_{m}$ can have a magnitude larger than $D_{j 1}$. To detect the first information-bearing signal, the output of $m$ th correlator can have two values and can be rewritten as

$$
\mathrm{D}_{\mathrm{m}}=\left\{\begin{array}{l}
\mathrm{SS}+\mathrm{SN}+\mathrm{NN} m=j_{1} \\
\mathrm{SI}+\mathrm{SN}+\mathrm{NN} m \neq j_{1}
\end{array}\right.
$$


The correlation components can be calculated as

$$
\begin{aligned}
& \mathrm{SS}=\mathrm{X}_{\beta} \mathrm{P}_{\mathrm{j}_{1}}{ }^{-1} \mathrm{P}_{\mathrm{j}_{1}} \mathrm{X}_{\beta}=\mathrm{X}_{\beta} \mathrm{X}_{\beta}{ }^{\mathrm{T}}=\sum_{\mathrm{k}=1}^{\beta} \mathrm{x}_{\mathrm{k}} \mathrm{x}_{\mathrm{k}} \\
& \mathrm{SN}=\mathrm{X}_{\beta} \mathrm{W}_{0}+\mathrm{W}_{\beta} \mathrm{P}_{\mathrm{j}_{1}}{ }^{-1} \mathrm{P}_{\mathrm{j}_{1}} \mathrm{X}=\sum_{\mathrm{k}=1}^{\beta} \mathrm{x}_{\mathrm{k}-\beta} \mathrm{w}_{\mathrm{k}}+\sum_{\mathrm{k}=1}^{\beta} \mathrm{w}_{\mathrm{k}-\beta} \mathrm{x}_{\mathrm{k}} \\
& \mathrm{NN}=\mathrm{W}_{\beta} \mathrm{P}_{\mathrm{j}_{1}}^{-1} \mathrm{~W}_{0}=\sum_{\mathrm{k}=1}^{\beta} \mathrm{w}_{\mathrm{k}-\beta} \mathrm{W}_{\mathrm{k}}^{\prime},
\end{aligned}
$$

where $w_{i}^{\prime}$ is the permutated noise sample.

Based on the assumption that the correlation between noise sample $w_{i}$ and chaotic samples $x_{j}$ for all $i$ and $j$ is negligible, for sufficient value of $\beta$, then

$$
\begin{aligned}
& E\left(D_{j_{1}}\right)=E(\mathrm{SS})+\mathrm{E}(\mathrm{SN})+\mathrm{E}(\mathrm{NN})=\mathrm{E}\left(\sum_{\mathrm{k}=1}^{\beta} \mathrm{x}_{\mathrm{k}} \mathrm{x}_{\mathrm{k}}\right)+0+0=\beta \mathrm{V}(\mathrm{x})=\frac{\mathrm{L}}{\mathrm{L}+1} \mathrm{nE}_{\mathrm{b}} \\
& V(\mathrm{SS})=\mathrm{V}\left(\sum_{\mathrm{k}=1}^{\beta} \mathrm{x}_{\mathrm{k}} \mathrm{x}_{\mathrm{k}}\right)=\mathrm{V}\left(\sum_{\mathrm{k}=1}^{\beta} \mathrm{x}^{2} \mathrm{k}\right)=\frac{4}{5} \beta \mathrm{V}(\mathrm{x}) \mathrm{V}(\mathrm{x})=\frac{4(\mathrm{~L})^{2}}{5 \beta(\mathrm{L}+1)^{2}} \mathrm{n}^{2} \mathrm{E}_{\mathrm{b}}^{2} \\
& V(\mathrm{SN})=2 \beta V(x) V(w)=2 \frac{L n}{L+1} E_{b} N_{0} \\
& V(\mathrm{NN})=V(w) . V\left(w^{\prime}\right)=\frac{\beta}{4} N_{o}^{2} .
\end{aligned}
$$

While $E\left(D_{m}\right) \approx 0$, similarly

$$
\begin{aligned}
& V\left(D_{m}\right)=V(\mathrm{SI})+\mathrm{V}(\mathrm{SN})+\mathrm{V}(\mathrm{NN}) \\
& =\frac{(L)^{2} n^{2}}{\beta(L+1)^{2}} E_{b}^{2}+2 \frac{L n}{(L+1)} E_{b} N_{0}+\frac{\beta}{4} N_{o}^{2}
\end{aligned}
$$

The output of each $2^{n}-1$ correlators $D_{m}$ for $m \neq j_{1}$ are statistically independent random values characterized by a normal distribution with zero mean and can be given as

$$
f_{D_{m}}(y)=\frac{1}{\sqrt{2 \pi V\left(D_{m}\right)}} e^{-\frac{(y)^{2}}{2 V\left(D_{m}\right)}}
$$

While the correlator output conditioned by correct permutation can be given as

$$
f_{D_{j_{1}}}(y)=\frac{1}{\sqrt{2 \pi V\left(D_{j_{1}}\right)}} e^{-\frac{\left(y-E\left(D_{j_{1}}\right)\right)^{2}}{2 V\left(D_{j_{1}}\right)}}
$$


Table 2 Transmitter system complexity analysis (for one information bits)

\begin{tabular}{llllll}
\hline Unit & DCSK & CDSK & HE-DCSK & TR-DCSK & SR-PI-DCSK \\
\hline Adders & 0 & 1 & 1 & 1 & 0 \\
Multipliers & 1 & 1 & 1 & 1 & 0 \\
Delay & 1 & 1 & 1 & 0 & L \\
Modulator & 1 & 1 & 1 & 1 & 0 \\
DPU & 0 & 0 & 0 & 1 & $L$ \\
\hline
\end{tabular}

${ }^{\mathrm{a}} D P U$ data permutation unit (including the time reversal unit)

Table 3 Receiver system complexity analysis (for one information bit)

\begin{tabular}{llllll}
\hline Unit & DCSK & CDSK & HE-DCSK & TR-DCSK & SR-PI-DCSK \\
\hline Multipliers & 1 & 1 & 1 & 0 & M/n \\
Delay & 1 & 1 & 1 & 1 & $\mathrm{~L} / \mathrm{n}$ \\
Demodulator & 1 & 1 & 1 & 1 & $\mathrm{M} / \mathrm{n}$ \\
DMU a & 0 & 0 & 0 & 1 & $1 / n$ \\
\hline
\end{tabular}

${ }^{\mathrm{a}} D M U$ data mapping unit (including the time reversal unit)

It is easier to calculate the probability of correct permutation which is occurred only if magnitude of $D j_{1}>D_{1}, D j_{1}>D_{2}$ and $\ldots D j_{1}>D_{M}$, therefore the probability of correct map detection can be given as [22]

$$
p_{\text {map }}=1-\int_{0}^{\infty} F_{D m}(y)^{2^{n}-1} f_{j_{1}}\left(D_{j_{1}}\right)(y) \mathrm{dy},
$$

where $F_{D m}(y)$ is the commutative distribution function given by

$$
F_{D_{m}}(y)=\operatorname{erf}\left(\frac{y}{\sqrt{2 \pi V\left(D_{m}\right)}}\right)
$$

The overall BER of the system can be given by substitution on

$$
\begin{aligned}
\text { BER }_{\text {SR-PI-DCSK }}= & \frac{2^{(n-1)}}{2^{n}-1}\left[1-\frac{1}{\sqrt{2 \pi \frac{4(L)^{2} n^{2}}{5 \beta(L+1)^{2}} E_{b}^{2}+2 \frac{L n}{L+1} E_{b} N_{0}+\frac{\beta}{4} N_{o}^{2}}}\right. \\
& \int_{0}^{\infty} \operatorname{erf}\left(\frac{y}{\sqrt{2 \pi \frac{(L)^{2} n^{2}}{\beta(L+1)^{2}} E_{b}^{2}+2 \frac{L n}{L+1} E_{b} N_{0}+\frac{\beta}{4} N_{o}^{2}}}\right)^{2^{n}-1} \\
& \left.\mathrm{e}^{-\frac{\left(\mathrm{y}-\mathrm{E}\left(\mathrm{D}_{j_{1}}\right)\right)^{2}}{2\left(_{\left.\mathbb{D}_{j_{1}}\right)}\right.}} \mathrm{dy}\right]
\end{aligned}
$$

When $L$ is very large, the overall BER of the system can be given by 


$$
\begin{aligned}
& \text { BER }_{\text {SR-PI-DCSK }}=\frac{2^{(n-1)}}{2^{n}-1}\left[1-\frac{1}{\sqrt{2 \pi \frac{4 n^{2}}{5 \beta} E_{b}^{2}+2 n E_{b} N_{0}+\frac{\beta}{4} N_{o}^{2}}}\right. \\
& \int_{0}^{\infty} \operatorname{erf}\left(\frac{y}{\sqrt{2 \pi \frac{n^{2}}{\beta} E_{b}^{2}+2 n E_{b} N_{0}+\frac{\beta}{4} N_{o}^{2}}}\right)^{2^{n}-1} \\
& \mathrm{e}^{-\frac{\left(\mathrm{y}-\mathrm{E}\left(\mathrm{D}_{\mathrm{j}_{1}}\right)\right)^{2}}{2 V\left(\mathbb{D}_{\mathfrak{j}_{1}}\right)}} \mathrm{dy} \\
&
\end{aligned}
$$

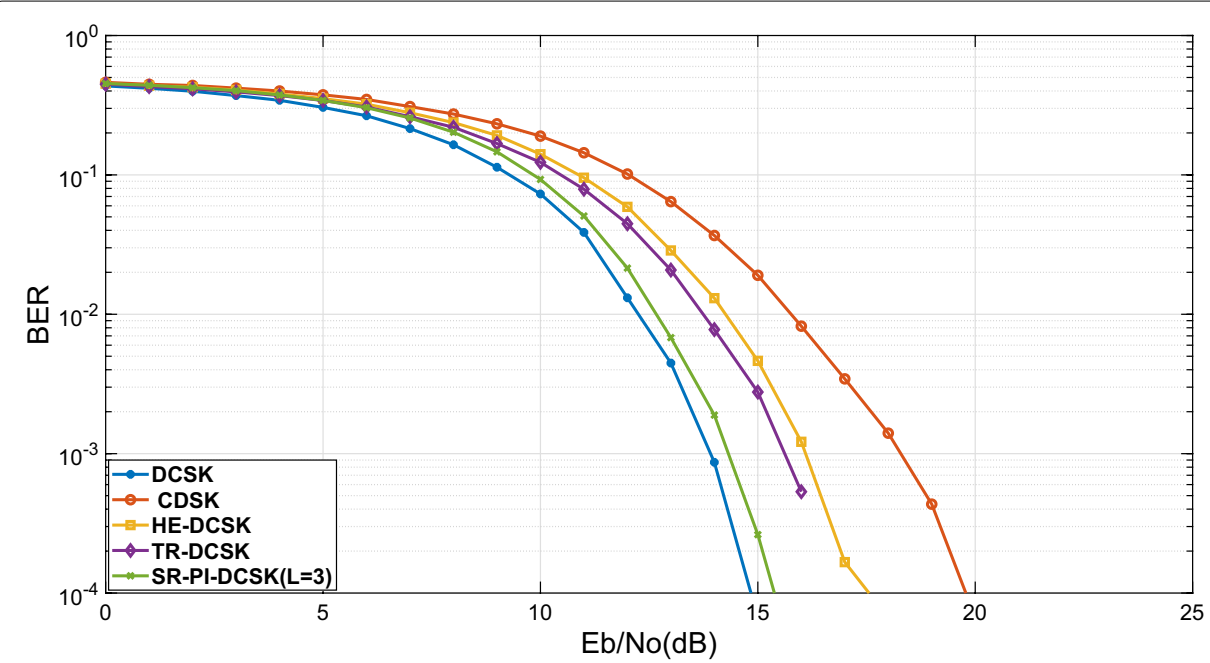

Fig. 4 The bit error rate performance of SR-PI-DCSK versus DCSK, CDSK, HE-DCSK, and TR-DCSK for $\beta=100$

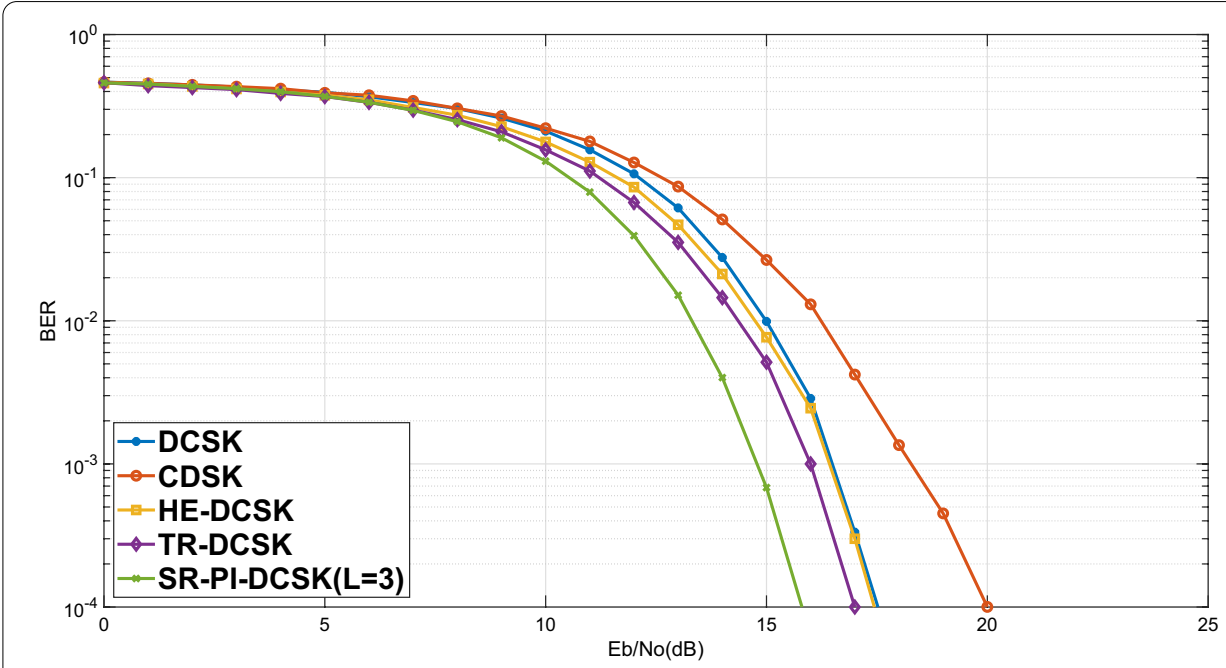

Fig. 5 The bit error rate performance of SR-PI-DCSK versus DCSK, CDSK, HE-DCSK, and TR-DCSK for $\beta=150$ 


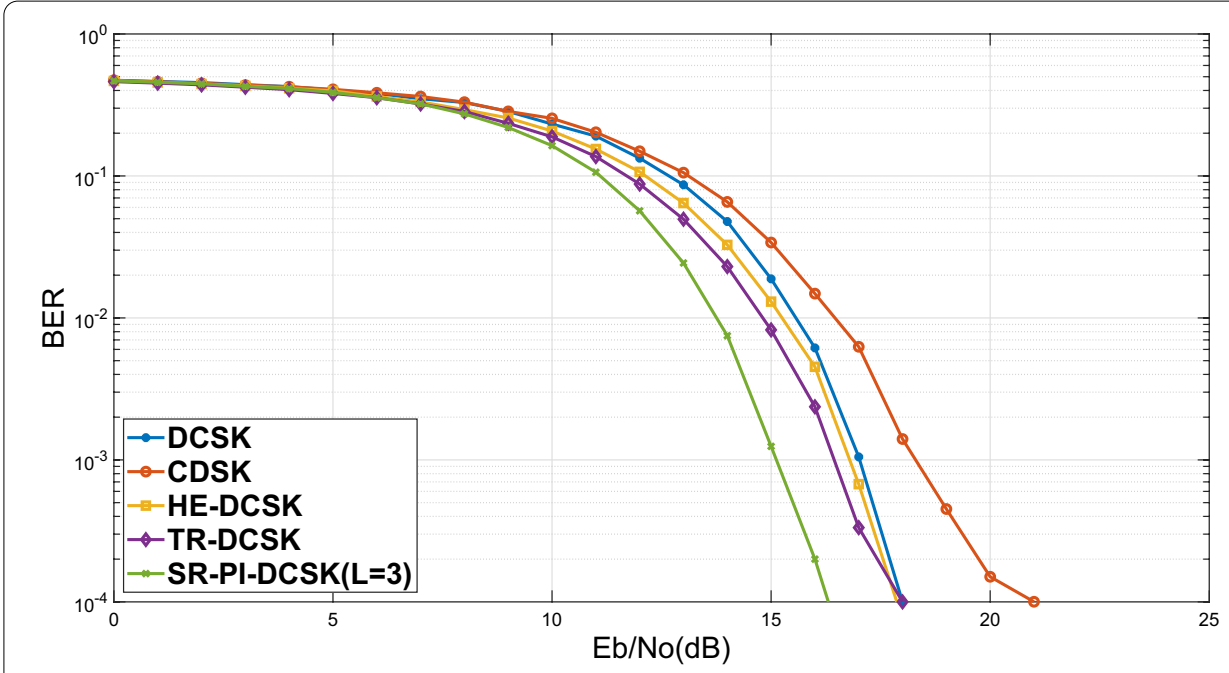

Fig. 6 The bit error rate performance of SR-PI-DCSK versus DCSK, CDSK, HE-DCSK, and TR-DCSK for $\beta=200$

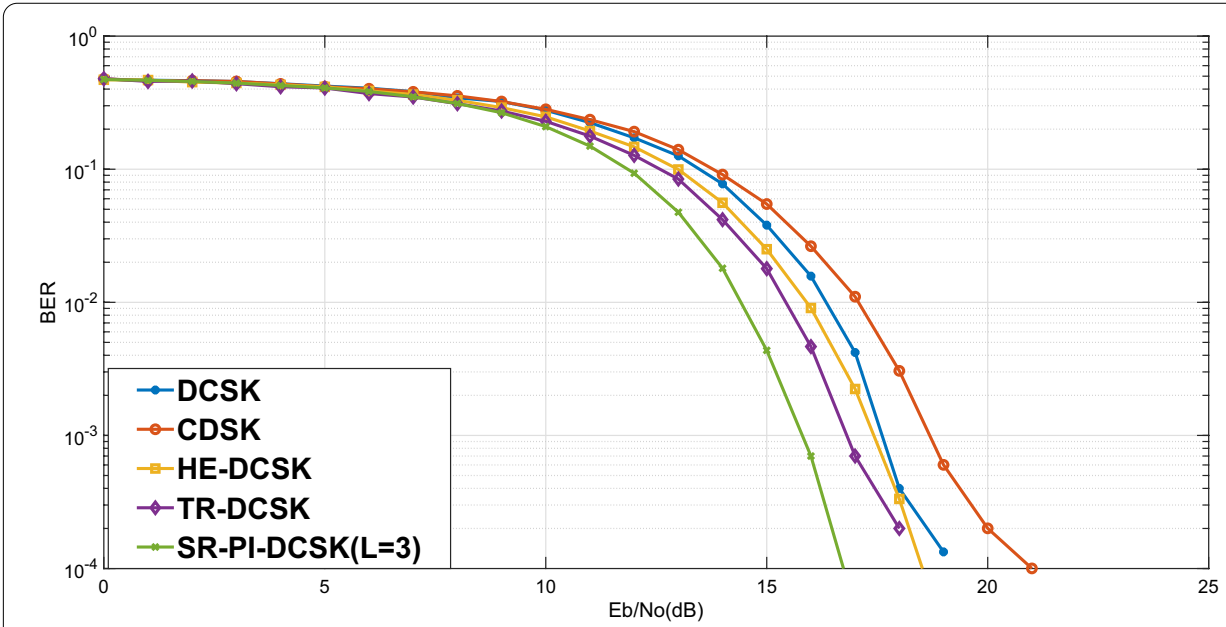

Fig. 7 The bit error rate performance of SR-PI-DCSK versus DCSK, CDSK, HE-DCSK, and TR-DCSK for $\beta=300$

Table 4 SR-PI-DCSK performance comparison with different chaotic systems at different spreading factors

\begin{tabular}{lllll}
\hline Spread factor $(\beta)$ & DCSK & CDSK & HE-DCSK & TR-DCSK \\
\hline 100 & Less performance & Outperform & Outperform & Outperform \\
150 & Outperform & Outperform & Outperform & Outperform \\
200 & Outperform & Outperform & Outperform & Outperform \\
300 & Outperform & Outperform & Outperform & Outperform \\
\hline
\end{tabular}




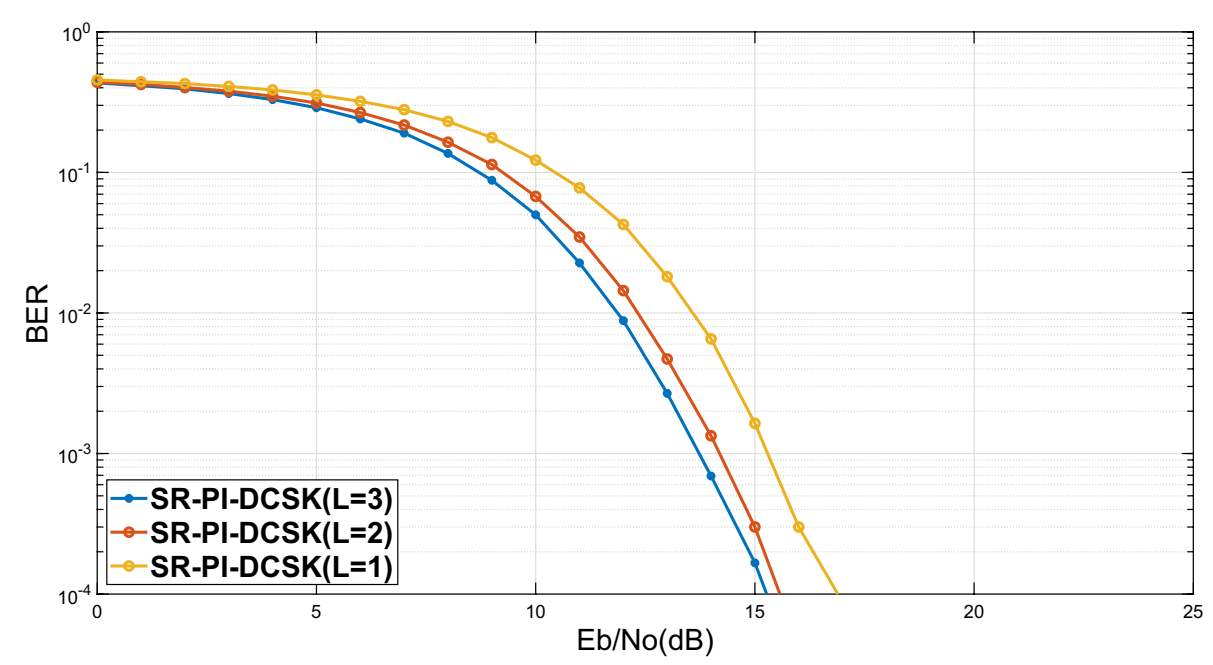

Fig. 8 The bit error rate performance at different frame lengths $(L=1, L=2$ and $L=3)$ for SR-PI-DCSK for $\beta=50$

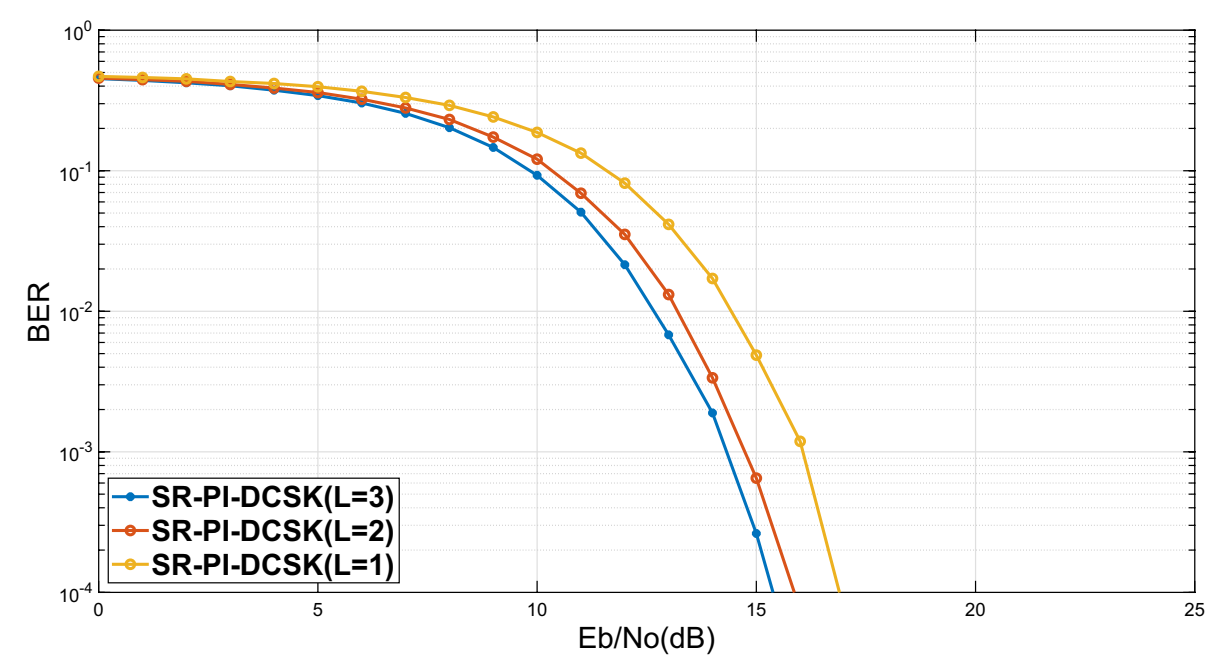

Fig. 9 The bit error rate performance at different frame lengths $(L=1, L=2$ and $L=3$ ) for SR-PI-DCSK for $\beta=100$

\section{Complexity analysis}

In this section, system complexity is evaluated against a number of essential components in each system. The evaluation is calculated based on the hardware resources required to transmit and receive single bits. Table 2 compares the transmitter of the proposed system with DCSK, CDSK, HE-DCSK, and TR-DCSK. The SR-PI-DCSK transmitter is less complex than CDSK, HE-DCK and TR-DCSK systems in terms of adder, multiplier 


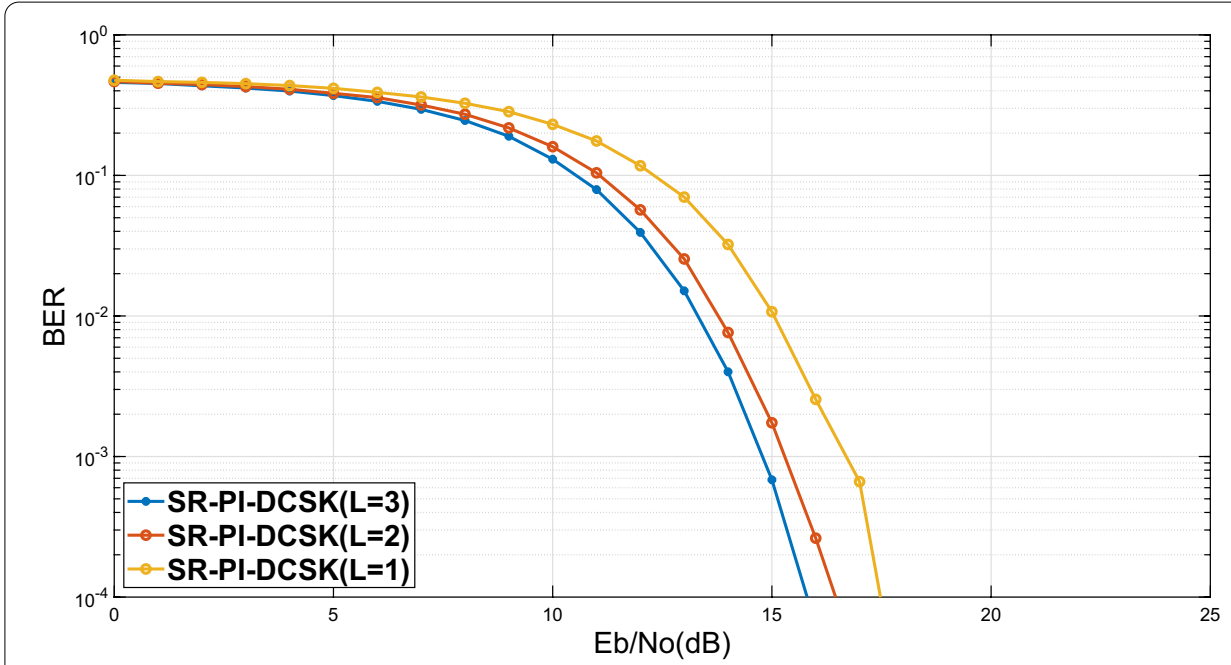

Fig. 10 The bit error rate performance at different frame lengths $(L=1, L=2$ and $L=3$ ) for SR-PI-DCSK for $\beta=150$

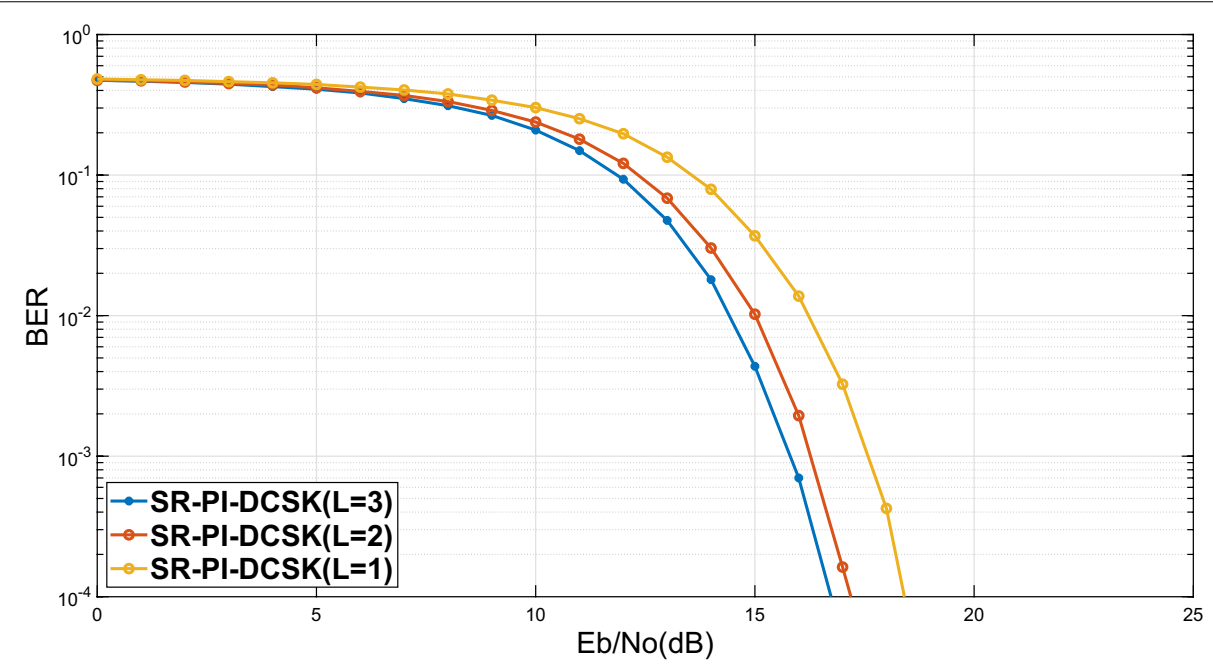

Fig. 11 The bit error rate performance at different frame lengths $(L=1, L=2$ and $L=3$ ) for SR-PI-DCSK for $\beta=300$

and modulator. However, the proposed system used delay and permutations units and required to transmit $L$ bits in each frame. The other systems required one bit delay and didn't have data permutation unit, except TR-DCSK that did not include delay unit and required one bit for data permutation.

The receiver of the proposed scheme requires $L / n$ delay element which helps to reuse the structure of receiver $L$ times as shown in Fig. 3. Detailed receiver complexity of the SR-PI-DCSK compared with other systems is illustrated in Table 3. The receiver of the proposed scheme is more complexity than other systems as it required more delay and inverse permutation functions. 


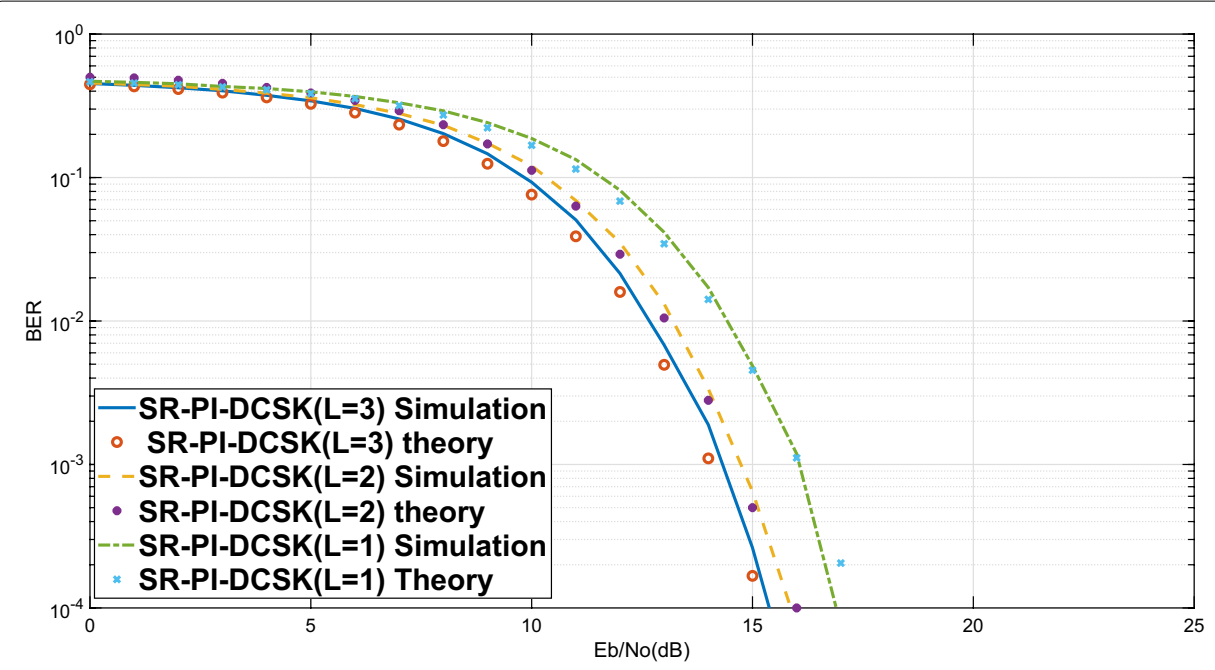

Fig. 12 Theoretical and simulation bit error performance for SR-PI-DCSK for different frame lengths for $\beta=100$. Simulation results are depicted by lines while theoretical results are depicted by markers

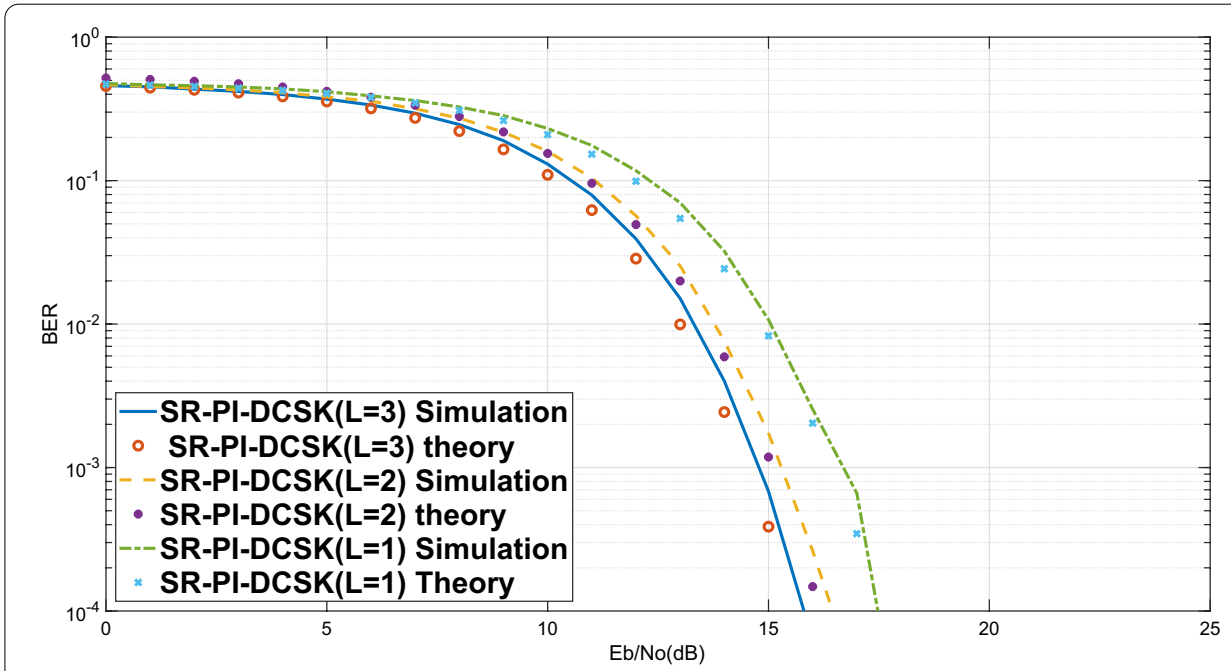

Fig. 13 Theoretical and simulation bit error performance for SR-PI-DCSK for different frame lengths for $\beta=150$. Simulation results are depicted by lines while theoretical results are depicted by markers

\section{Experimental results (theoretical and simulation)}

In this section, different standard chaos schemes including the recently proposed scheme have been simulated and compared with the suggested system in AWGN channel environment and with various $E_{b} / N_{0}$. Furthermore, the proposed system has been compared with the standard PI-DCSK [19]. Analytic performance based on Eq. 24 has been compared with simulation results for different frame lengths. Finally, the effect of the spreading factors on the proposed scheme has been examined.

In Fig. 4, the SR-PI-DCSK is compared with the DCSK, CDSK, HE-DCSK, and TRDCSK. The system outperforms the other systems by an average of $2 \mathrm{~dB}$ except for DCSK which exceeds the suggested system by $1 \mathrm{~dB}$, although the proposed system 


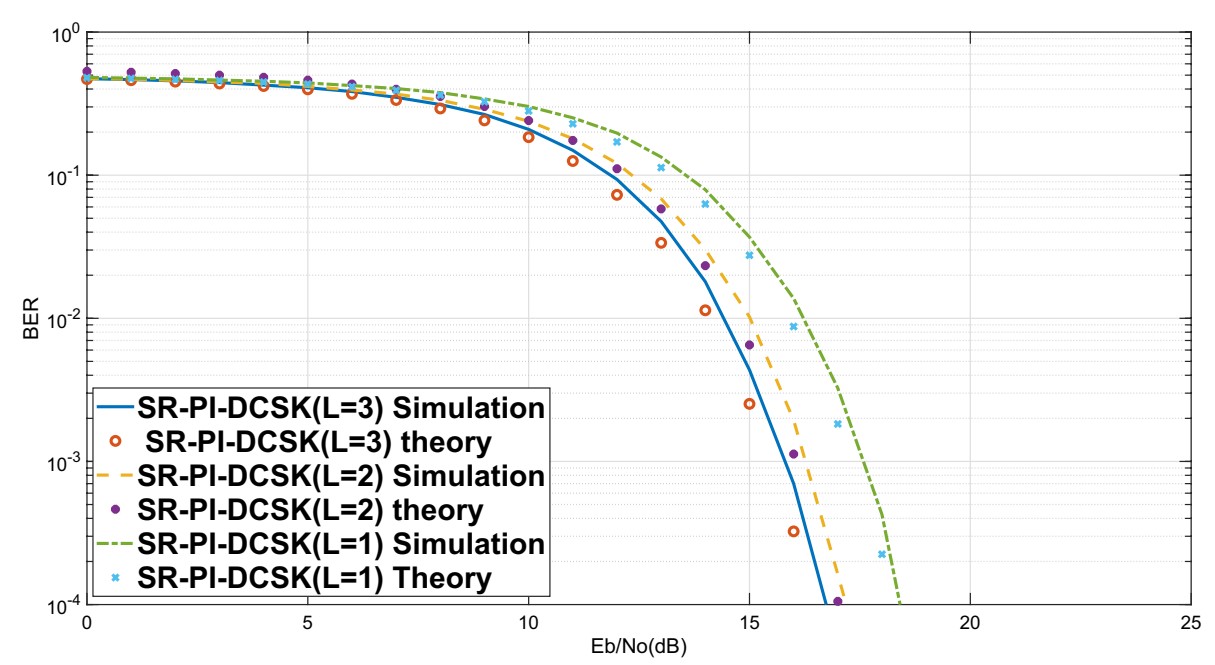

Fig. 14 Theoretical and simulation bit error performance for SR-PI-DCSK for different frame lengths for $\beta=300$. Simulation results are depicted by lines while theoretical results are depicted by markers

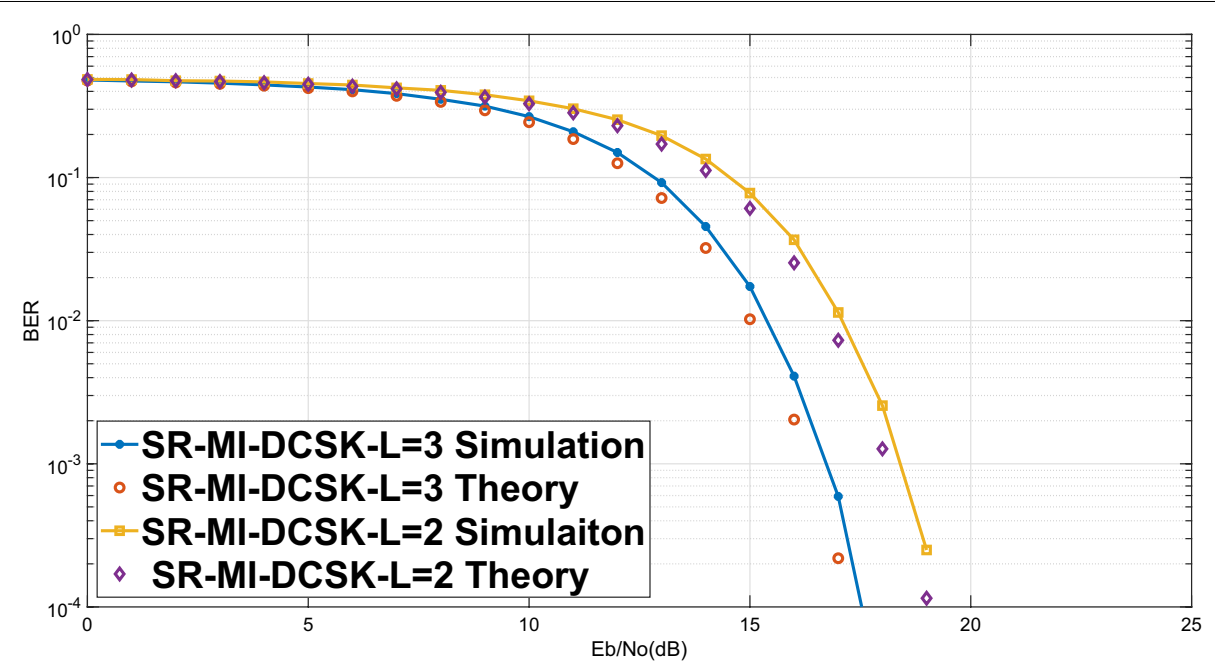

Fig. 15 Theoretical and simulation bit error performance for SR-PI-DCSK for different frame lengths for $\beta=500$. Simulation results are depicted by lines while theoretical results are depicted by markers

offers a double bit rate. This is due to non-complete orthogonality at spreading factor $\beta=100$. When the spreading factor is increased to $\beta=150$, it can be easily noted that the SR-PI-DCSK system performance is enhanced by an average of $3 \mathrm{~dB}$ for all other systems as shown in Fig. 5. Obviously, the superiority in the performance continued while spreading factor increased to $\beta=200$ and $\beta=300$ as shown in Figs. 6 and 7, respectively. This can be explained by the fact that, compared to other system chaos signal, the average transmitted signal energy per bit for the proposed system has been saved by $2 L /(L+1) \approx 50 \%$ compared to the standard systems to which corresponds to a gain or horizontal displacement of BER. In addition to the very low cross-correlation value at the correlator output between the interference component. 


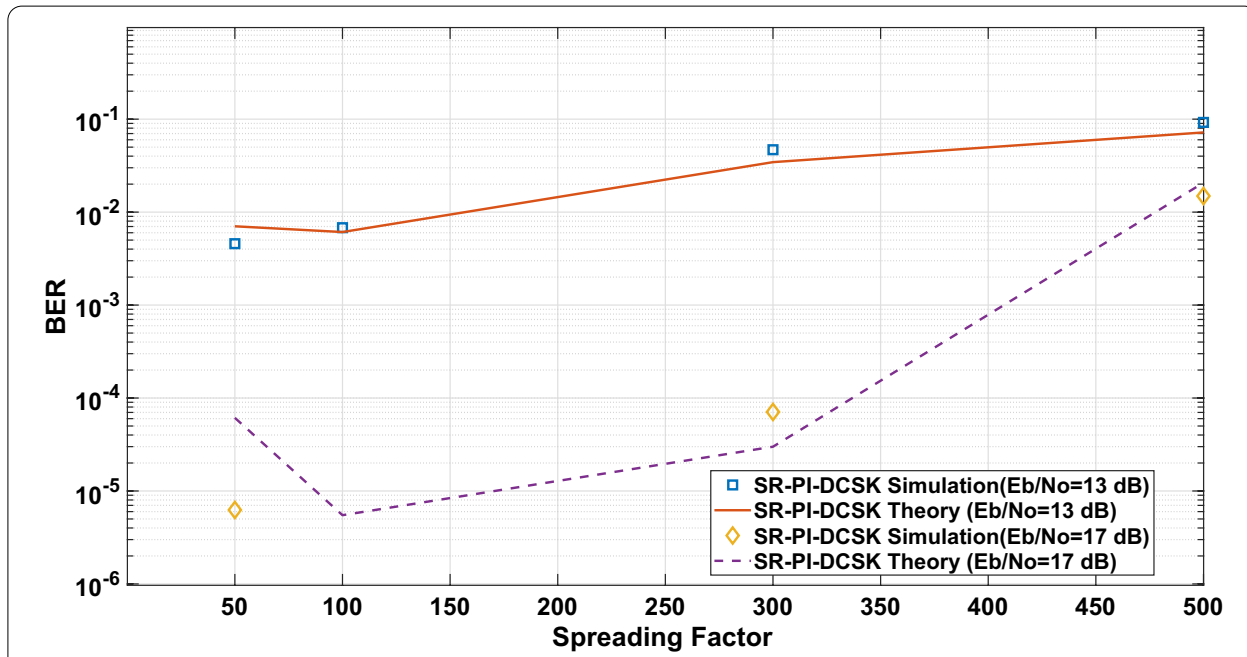

Fig. 16 Theoretical and simulation bit error performance for SR-PI-DCSK at different spreading values and $L=3$

Table 4 shows summary of the performance of the SR-PI-DCSK, the proposed system outperform all others systems except DCSK at $\beta=100$.

Comparison of the SR-PI-DCSK system performance at various frame lengths is demonstrated in Figs. 8, 9, 10 and 11. The comparison is performed at the increment trend of the spreading factor. It is obvious that the performance of the proposed system with frame length $L=3$ is better than systems with the length of $L=2$ and $L=1$ or PI-DCSK [19]. It is evident that the performance is degraded at a high value of $\beta$ due to noise to noise (NN on Eq. 12) which at the correlator output.

A theoretical estimation of BER for the proposed system is developed using GA and validated using computer simulation. Excellent agreement between theoretical expression in Eq. 24 and simulation results that shown in Figs. 12, 13, 14 and 15 for $\beta=100, \beta=150, \beta=300$ and $\beta=500$, respectively. The results obtained clearly validate the expression derived.

Effect of increasing of spread factor $\beta$ has been studied and is illustrated in Fig. 16 at fixed value of $E_{b} / N_{0}$ (at $13 \mathrm{~dB}$ and $17 \mathrm{~dB}$ ). It is clearly shown that the performance is enhanced as $\beta$ increases and reaches its ideal performance at $\beta=100$, and this matches with the results shown in Figs. 4, 5, 6, and 7. After $\beta$ reaching 100, the performance starts to decrease with $\beta$ due to dominance of noise to noise expression $(N N)$ at high spreading factor as illustrated by Eq. (24).

\section{Conclusion}

A new permutation-based DCSK system named single reference-permutation indexdifferential chaos shift keying (SR-PI-DCSK) is proposed, designed, and validated through simulation. A theoretical formula to predict the bit error rate is developed and verified. The proposed system sends information symbols in a frame; each frame consists of a reference signal followed by a sequence of $L$ information-bearing signal. This reduces the energy requirement and enhances the BER. The BER performance is simulated and compared with DCSK, CDSK, HE-DCSK, and PI-DCSK schemes. 
Results illustrate the system outperform other chaos-based systems, particularly at large spreading factor. Comparison results illustrate the advantage of the proposed scheme specifically at moderate $E_{b} / N_{0}$ over the other schemes. Based on the GA method, theoretical prediction for BER is developed and compared with the simulation results. Excellent matching between the derived form and simulation results is noticed at typical values of spread factor.

\section{Abbreviations}

AWGN: Additive white Gaussian noise; BER: Bit error rate; CDSK: Correlation delay shift keying; CSK: Chaos shift keying; DCSK: Differential chaos shift keying; GA: Gaussian approximation; HE-DCSK: High efficiency-DCSK; MC-DCSK: Multicarrier modulation-DCSK; RF: Radio frequency; SR-PI-DCSK: Single reference-permutation index-differential chaos shift keying; STBC-DCSK: Space-time block code DCSK; PDF: Probability density function; PI-DCSK: Permuted index-differential chaos shift keying; TR-DCSK: Time reversal-DCSK

\section{Acknowledgements}

No acknowledgment to be considered.

\section{Authors' contributions}

Al Bassam conceived of the presented the SR-PI-DCSK scheme, developed the theoretical model and performed the mathematical analysis and simulation Al-Jerew performed and validated the simulation results with the theoretical model, verified result consistency and contributed to the research literature section. Both authors discussed the results and contributed to the final manuscript. Both authors read and approved the final manuscript.

\section{Funding}

The authors are responsible to provide the required funding.

\section{Availability of data and materials}

All data and materials are generated based on simulation.

\section{Declarations}

Competing interests

The authors declare that they have no competing interests. The authors declare that they have no competing interests.

\section{Author details}

${ }^{1}$ Department of Electronics and Communications Engineering, Middle East College, Muscat, Oman. ${ }^{2}$ Business Information Systems, Asia Pacific International College, Sydney, Australia.

Received: 6 March 2021 Accepted: 12 October 2021

Published online: 27 November 2021

\section{References}

1. U. Parlitz, L.O. Chua, L. Kocarev, K. Halle, A. Shang, Transmission of digital signals by chaotic synchronization. Int. J. Bifurc. Chaos 2(04), 973-977 (1992)

2. G. Kolumbán, M.P. Kennedy, L.O. Chua, The role of synchronization in digital communications using chaos. II. Chaotic modulation and chaotic synchronization. IEEE Trans. Circuits Syst. I Fundam. Theory Appl. 45(11), 1129-1140 (1998)

3. M. Sushchik, L.S. Tsimring, A.R. Volkovskii, Performance analysis of correlation-based communication schemes utilizing chaos. IEEE Trans. Circuits Syst. I Fundam. Theory Appl. 47(12), 1684-1691 (2000)

4. A.J. Lawrance, J. Yao, Likelihood-based demodulation in multi-user chaos shift keying communication. Circuits Syst. Signal Process. 27(6), 847-864 (2008)

5. M. Long, Y. Chen, F. Peng, Bit error rate improvement for chaos shift keying chaotic communication systems. IET Commun. 6(16), 2639-2644 (2012)

6. G. Kis, Z. Jako, M. Kennedy, G. Kolumbán, Chaotic communications without synchronization (1998)

7. C.K. Tse, F.C. Lau, A return map regression approach for noncoherent detection in chaotic digital communications. Int. J. Bifurc. Chaos 13(03), 685-690 (2003)

8. G. Kolumbán, Z. Jákó, M.P. Kennedy, Enhanced versions of DCSK and FM-DCSK data transmission systems. In: ISCAS'99. Proceedings of the 1999 IEEE International Symposium on Circuits and Systems VLSI (Cat. No. 99CH36349), vol. 4, pp. 475-478 (1999). IEEE

9. W.M. Tam, F.C.-M. Lau, C. Tse, A multiple access scheme for chaos-based digital communication systems utilizing transmitted reference. IEEE Trans. Circuits Syst. I Regul. Pap. 51(9), 1868-1878 (2004)

10. H. Yang, G.-P. Jiang, High-efficiency differential-chaos-shift-keying scheme for chaos-based noncoherent communication. IEEE Trans. Circuits Syst. II Express Briefs 59(5), 312-316 (2012) 
11. H. Yang, G.P. Jiang, Reference-modulated DCSK: a novel chaotic communication scheme. IEEE Trans. Circuits Syst. II Express Briefs 60(4), 232-236 (2013)

12. W. Xu, L. Wang, G. Kolumbán, A novel differential chaos shift keying modulation scheme. Int. J. Bifurc. Chaos 21 (03), 799-814 (2011)

13. G. Kaddoum, F. Gagnon, Design of a high-data-rate differential chaos-shift keying system. IEEE Trans. Circuits Syst. II Express Briefs 59(7), 448-452 (2012)

14. M. Xu, H. Leung, A novel high data rate modulation scheme based on chaotic signal separation. IEEE Trans. Commun. 58(10), 2855-2860 (2010)

15. G. Kaddoum, F.-D. Richardson, F. Gagnon, Design and analysis of a multi-carrier differential chaos shift keying communication system. IEEE Trans. Commun. 61(8), 3281-3291 (2013)

16. P. Chen, L. Wang, F.C. Lau, One analog STBC-DCSK transmission scheme not requiring channel state information. IEEE Trans. Circuits Syst. I Regul. Pap. 60(4), 1027-1037 (2013)

17. F.C. Lau, K.Y. Cheong, C. Tse, Permutation-based DCSK and multiple-access DCSK systems. IEEE Trans. Circuits Syst. I Fundam. Theory Appl. 50(6), 733-742 (2003)

18. K.Y. Cheong, F.C. Lau, K.T. Chi, Permutation-based M-ary chaotic-sequence spread-spectrum communication systems. Circuits Syst. Signal Process. 22(6), 567-577 (2003)

19. M. Herceg, G. Kaddoum, D. Vranješ, E. Soujeri, Permutation index DCSK modulation technique for secure multiuser high-data-rate communication systems. IEEE Trans. Veh. Technol. 67(4), 2997-3011 (2017)

20. M. Herceg, D. Vranješ, G. Kaddoum, E. Soujeri, Commutation code index DCSK modulation technique for high-datarate communication systems. IEEE Trans. Circuits Syst. II Express Br. 65(12), 1954-1958 (2018)

21. A. Dukhan, D. Jayalath, P. van Heijster, B. Senadji, J. Banks, A generalized multilevel-hybrid chaotic oscillator for lowcost and power-efficient short-range chaotic communication systems. EURASIP J. Wirel. Commun. Netw. 2020(1), $1-14(2020)$

22. W. Xu, Y.Tan, F.C. Lau, G. Kolumbán, Design and optimization of differential chaos shift keying scheme with code index modulation. IEEE Trans. Commun. 66(5), 1970-1980 (2018)

23. X. Cai, W. Xu, S. Hong, L. Wang, Dual-mode differential chaos shift keying with index modulation. IEEE Trans. Commun. 67(9), 6099-6111 (2019)

24. G. Zhang, X. Li, X. Chen, H. Xing, The m-ary security differential chaotic frequency shift system based on the MIMO model. Telecommun. Radio Eng. 79(5), 413-424 (2020)

25. H.A. Abdullah, H.N. Abdullah, W.A. Mahmoud Al-Jawher, A hybrid chaotic map for communication security applications. Int. J. Commun. Syst. 33(4), 4236 (2020)

26. N. Nguyen, L. Pham-Nguyen, M.B. Nguyen, G. Kaddoum, A low power circuit design for chaos-key based data encryption. IEEE Access 8, 104432-104444 (2020)

27. Y. Guo, J. Yang, B. Liu, Application of chaotic encryption algorithm based on variable parameters in RFID security. EURASIP J. Wirel. Commun. Netw. 2021(1), 1-17 (2021)

28. C. Liang, Q. Zhang, J. Ma, K. Li, Research on neural network chaotic encryption algorithm in wireless network security communication. EURASIP J. Wirel. Commun. Netw. 2019(1), 1-10 (2019)

29. T. Li, W. Yan, L. Ping, P. Fang, A WSN positioning algorithm based on 3 D discrete chaotic mapping. EURASIP J. Wirel. Commun. Netw. 2019(1), 1-13 (2019)

30. L. Novosel, G. Šišul, Performance evaluation of chaotic spreading sequences on software-defined radio. EURASIP J. Wirel. Commun. Netw. 2017(1), 1-12 (2017)

31. J.R. Pulido-Luna, J.A. López-Rentería, N.R. Cazarez-Castro, Design of a nonhomogeneous nonlinear synchronizer and its implementation in reconfigurable hardware. Math. Comput. Appl. 25(3), 51 (2020)

32. N.X. Quyen, Ber analysis for $2 \times 2$ mimo high-efficiency DCSK system. Electrica 21(1), 50-58 (2021)

33. G. Kaddoum, Wireless chaos-based communication systems: a comprehensive survey. IEEE Access 4, 2621-2648 (2016)

\section{Publisher's Note}

Springer Nature remains neutral with regard to jurisdictional claims in published maps and institutional affiliations.

\section{Submit your manuscript to a SpringerOpen ${ }^{\circ}$ journal and benefit from:}

- Convenient online submission

- Rigorous peer review

- Open access: articles freely available online

- High visibility within the field

- Retaining the copyright to your article

Submit your next manuscript at $\boldsymbol{\nabla}$ springeropen.com 